\title{
IDENTIFICAÇÃO DE SUBSTÂNCIAS EM ANÁLISE TOXICOLÓGICA SISTEMÁTICA UTILIZANDO UM SISTEMA INFORMATIZADO PARA CÁLCULO DE PARÂMETROS CROMATOGRÁFICOS E BUSCA EM BASES DE DADOS
}

\author{
Rafael Linden* e Sander Sartori \\ Curso de Ciências Farmacêuticas, Centro Universitário Feevale, Rodovia RS 239, 2755, 93352-000 Novo Hamburgo - RS, \\ Brasil \\ Estefânio Kellermann \\ Centro de Sistemas de Informação, Centro Universitário Feevale, Rodovia RS 239, 2755, 93352-000 Novo Hamburgo - RS, \\ Brasil \\ André Arigony Souto \\ Faculdade de Química, Pontifícia Universidade Católica do Rio Grande do Sul, Av. Ipiranga, 6681, 90619-900 Porto Alegre - \\ RS, Brasil
}

Recebido em 16/11/05; aceito em 9/5/06; publicado na web em 31/10/06

\begin{abstract}
SUBSTANCE IDENTIFICATION IN SYSTEMATIC TOXICOLOGICAL ANALYSIS USING A COMPUTER SYSTEM FOR CHROMATOGRAPHIC PARAMETER CALCULATION AND DATABASE RETRIEVAL. In spite of the availability of large databases of chromatographic data on several standardized systems, one major task in systematic toxicological analysis remains, namely how to handle the experimental data and retrieve data from the large available databases in a meaningful and productive way. To achieve this purpose, our group proposed an Internet-based tool using previously published STA databases, which interlaboratorial reproducibility tests have already evaluated. The developed software has the capability to calculate corrected chromatographic parameters, after the input of data obtained with standard mixtures of calibrators, and search the databases, currently incorporating TLC, color reactions, GC and HPLC data. At the end of the process, a list with candidate substances and their similarity indexes is presented.
\end{abstract}

Keywords: systematic toxicological analysis; database retrieval; chromatography.

\section{INTRODUÇÃO}

A identificação de substâncias de interesse toxicológico em amostras biológicas representa um desafio significativo, considerando a grande e crescente quantidade de substâncias potencialmente presentes, bem como a complexidade das matrizes e, freqüentemente, a disponibilidade de quantidades limitadas de amostra. Esse problema torna-se especialmente complexo quando se tem pouca ou nenhuma informação sobre o histórico do paciente ou da amostra, situação comum na toxicologia clínica e forense. Desta forma, este tipo de análise requer uma abordagem concisa e planejada, denominada Análise Toxicológica Sistemática (ATS) ${ }^{1,2}$.

A ATS pode ser definida como a busca químico-analítica em diferentes matrizes (sangue, plasma, tecidos) por substâncias potencialmente tóxicas, cuja presença é incerta e sua identidade é desconhecida ${ }^{1-4}$. O objetivo final da ATS é a exclusão de todas as substâncias potencialmente contidas na amostra, com exceção das efetivamente presentes. Ou seja, a identificação positiva ocorre quando os dados analíticos são compatíveis com uma determinada substância e incompatíveis com todas as demais substâncias possivelmente presentes na amostra, tais como metabólitos, compostos endógenos e demais interferentes ${ }^{5}$.

A ATS possui três fases, a saber: preparação da amostra, isolamento e concentração dos analitos; detecção e diferenciação e, por fïm, identificação ${ }^{1,2}$.

A identificação de substâncias em ATS é realizada através da comparação do seu comportamento em um determinado sistema analítico, estritamente padronizado, com dados presentes em bases de dados cuja reprodutibilidade interlaboratorial é conhecida. Consi-

*e-mail: rafael.linden@ feevale.br derando o grande número de analitos que potencialmente devem ser identificados em uma análise de triagem toxicológica (fármacos e metabólitos, praguicidas, produtos químicos de uso industrial e outros), que pode chegar a vários milhares de substâncias, é improvável que laboratórios individuais criem e mantenham bases de dados suficientemente amplas ${ }^{1,2,5,6}$. Desta forma, a disponibilidade de bases de dados aplicáveis interlaboratorialmente é fundamental em análise toxicológica sistemática.

Apesar da disponibilidade de métodos analíticos modernos de elevada sensibilidade e especificidade, tais como a cromatografia líquida associada à espectrometria de massas em tandem (CL-EM/ $\mathrm{EM})^{7,8}$ e espectrometria de massas por tempo de vôo (CL-TDV) ${ }^{9}$, a maior parte dos laboratórios de toxicologia analítica ainda utilizam métodos clássicos, como a cromatografia em camada delgada (CCD), cromatografia gasosa (CG) com detectores não espectrométricos, como os de ionização em chama (DIC) e nitrogênio-fósforo (DNP), e a cromatografia líquida de alta eficiência com detecção por absorção de radiação ultravioleta (CLAE-UV) $)^{2,5,6}$.

Considerando a necessidade da existência de sistemas de ATS robustos associados a amplas bases de dados de comportamento analítico, o comitê de análise toxicológica sistemática da "International Association of Forensic Toxicologists" (TIAFT) fomentou estudos de padronização de diversas metodologias para utilização interlaboratorial em ATS, bem como a geração de amplas bases de dados. Como resultado deste trabalho foram publicadas bases de dados com métodos e dados analíticos utilizando CCD e $\mathrm{CG}^{3,4}$. Posteriormente, diversos autores propuseram metodologias de ATS utilizando CLAE, especialmente com detectores de arranjo de diodos (DAD) ${ }^{10-22}$.

$\mathrm{Na}$ identificação toxicológica baseada em bases de dados, devese levar em consideração que somente compostos cujos dados estejam inseridos na base em utilização poderão ser identificados. Tam- 
bém, considerando o grau de especificidade de cada método, bem como a existência de variabilidades interlaboratoriais, representadas pelos desvios-padrões interlaboratoriais de cada sistema analítico, é necessária a associação de dados obtidos em diferentes sistemas para uma identificação efetiva. Também deve se considerar que o número de sistemas necessários para identificação inequívoca aumenta com o número de sustâncias presentes na população tomada em consideração ${ }^{1,2}$.

Apesar da existência de sistemas altamente reprodutíveis do ponto de vista interlaboratorial, o manuseio dos dados obtidos é problemático. Embora a base teórica da identificação de compostos através da busca em bases de dados esteja estabelecida ${ }^{1-4}$, sua implementação prática apresenta dificuldades. Especialmente, a busca manual em diferentes listas de substâncias-candidato, quando vários métodos são associados a uma mesma amostra, não é produtiva.

Com o propósito de permitir a utilização dos sistemas padronizados de ATS já existentes foi proposto um sistema informatizado, acessível através da internet, para cálculo de parâmetros cromatográficos, busca em base de dados e cálculo de índices de similaridade em análise toxicológica sistemática. Cabe destacar que atualmente não está disponível outro sistema com estas características.

\section{AVALIAÇÃO ESTATÍSTICA DOS SISTEMAS EMPREGADOS EM ATS}

Um método de identificação em ATS pode ser avaliado por diferentes parâmetros estatísticos, como o comprimento médio da lista $(\mathrm{CML})$ e o poder discriminante $(\mathrm{PD})^{3,23,24}$.

O poder discriminante (PD) é definido como a probabilidade de que duas substâncias selecionadas aleatoriamente possam ser separadas pelo sistema empregado. Se os parâmetros de retenção estão dentro de uma determinada janela de erro, eles são considerados indiscriminados ou agrupados e o conjunto de agrupamentos para todas as substâncias no sistema é denominado $\mathrm{M}$. O número de agrupamentos possíveis é $\mathrm{N}(\mathrm{N}-1) / 2$, onde $\mathrm{N}$ é o número total de substâncias no sistema. Então, a probabilidade de dois fármacos selecionados aleatoriamente estarem agrupados é $2 \mathrm{M} / \mathrm{N}(\mathrm{N}-1)^{3,23,24}$. O poder discriminante é expresso pela Equação 1.

$$
P D=1-(2 M / N(N-1))
$$

No cálculo de PD, assume-se que os dados obtidos em um procedimento laboratorial para uma determinada substância apresentam flutuações inter-dias e inter-laboratórios e que estas flutuações seguem um determinado padrão. Assume-se, também, que o parâmetro de retenção avaliado segue uma distribuição normal com referência ao valor médio listado na base de dados previamente construída e que possui um desvio-padrão determinado, dependente daquele sistema. Quanto maior a diferença entre o valor do parâmetro avaliado com aquele da base de dados, menor será a probabilidade de identificação positiva. Após uma normalização de tal forma que a soma das probabilidades seja 1, as substâncias podem ser ordenadas em uma ordem decrescente de probabilidade ${ }^{3}$.

O conceito do comprimento médio da lista (CML) foi apresentado em 1983, por Schepers e colaboradores ${ }^{23}$. Nesta abordagem estatística, é estabelecido o número de substâncias que são qualificáveis para um determinado parâmetro em um dado sistema. O número de substâncias qualificadas é chamado de comprimento da lista. O CML também pode ser determinado para combinações de sistemas. O ótimo é obtido quando obtém-se um CML de 1. Para o cálculo do CML, assume-se que os valores numéricos de qualquer parâmetro de identificação, medidos diversas vezes, seguem uma distribuição normal. Quando um certo limite de probabilidade é estabelecido, todos os padrões que se qualificam formam uma lista. Quanto menor esta lista, mais poderoso é o sistema de identificação ${ }^{23,24}$. A partir da média dos valores de comprimento de lista, calcula-se o CML segundo a Equação 2.

$$
C M L=\sum_{i=1}^{n} C L_{i} /
$$

onde: CL é o comprimento da lista para cada composto e n é o número de compostos da base de dados.

Quanto menor o CML, mais adequado para ATS é o sistema avaliado. O cálculo pode ser realizado para sistemas individuais bem como para combinação de sistemas, incluindo modos de detecção. O sistema analítico ideal possui um CML de 1, que significa que todas as substâncias de uma população são identificadas inequivocamente ${ }^{2}$.

\section{DETECÇÃO E DIFERENCIAÇÃO POR CROMATOGRAFIA EM CAMADA DELGADA}

A cromatografia em camada delgada é uma técnica popular para identificação de substâncias em toxicologia analítica devido à sua velocidade, confiabilidade e baixo custo. Além de permitir a separação dos compostos, através da variação tanto da fase estacionária quanto da fase móvel, a facilidade de aplicação de diferentes reagentes cromogênicos proporciona a obtenção de dados adicionais.

O parâmetro de identificação usual em CCD é o fator de retardamento $\left(R_{\mathrm{f}}\right)$, o qual é frequientemente expresso multiplicado por 100 $\left(h R_{f}\right)$. Os valores de $h R_{f}$ são influenciados por diversos fatores, tais como temperatura e umidade ambiental, tamanho da placa, saturação da cuba cromatográfica e quantidade de amostra aplicada. Com o propósito de minimizar a influência das condições ambientais nos valores do parâmetro de identificação em CCD, as bases de dados empregadas utilizam o $\mathrm{hR}_{\mathrm{f}}$ corrigido $\left(\mathrm{hR}_{\mathrm{f}} \mathrm{c}\right)$, parâmetro de identificação proposto inicialmente por Galanos e Kapoulas ${ }^{25}$. Diferentemente do fator de retardamento relativo $\left(\mathrm{hR}_{\mathrm{rel}}\right)$, o $\mathrm{hR}_{\mathrm{f}} \mathrm{c}$ é calculado tomando-se duas substâncias como referências. Os valores de $h_{\mathrm{f}} \mathrm{c}$ tem se mostrado extremamente estáveis interlaboratorialmente, mesmo em condições climáticas muito diversas ${ }^{3,26-29}$.

$\mathrm{O} \mathrm{hR}_{\mathrm{f}} \mathrm{c}$ é obtido ao analisar uma amostra da substância desconhecida, em qualquer um dos sistemas de CCD propostos, juntamente com o conjunto adequado de marcadores. A partir dos valores obtidos de $\mathrm{R}_{\mathrm{f}}$ para a substância desconhecida e para os marcadores e dos valores tabelados de $\mathrm{hR}_{\mathrm{f}} \mathrm{c}$ para os marcadores, pode-se calcular o valor de $\mathrm{hR}_{\mathrm{f}} \mathrm{c}$ do desconhecido, conforme a Equação $3^{1-3}$.

$h R_{f} c(X)=h R_{f} c(A)+\frac{\left[h R_{f} c(B)-h R_{f} c(A)\right]}{\left[h R_{f}(B)-h R_{f}(A)\right]} \bullet\left[h R_{f}(X)-h R_{f}(A)\right]$

onde: $\mathrm{X}$ refere-se à substância desconhecida e A e $\mathrm{B}$, aos padrões que eluem imediatamente antes e depois do desconhecido $\mathrm{e}_{\mathrm{f}}$ é o fator de retardamento.

A TIAFT publicou uma base de dados com valores de $h R_{f} c$ para substâncias de interesse toxicológico em 10 sistemas de CCD, apresentados na Tabela $1^{1-3}$.

Em um enfoque probabilístico, todas as substâncias presentes na base de dados com valor do parâmetro de identificação dentro de uma faixa ao redor do valor de $\mathrm{hR}_{\mathrm{f}} \mathrm{c}$ da substância desconhecida são potenciais candidatos, com probabilidades menores à medida que o valor tabelado se afasta do $\mathrm{hR}_{\mathrm{f}} \mathrm{c}$ do composto desconhecido. Usualmente, leva-se em consideração todas as substâncias na faixa que compreende três vezes o valor do desvio-padrão interlaboratorial associado às medidas do sistema analítico utilizado, denominada janela de busca ${ }^{5}$. 
Tabela 1. Sistemas padronizados para ATS por cromatografia em camada delgada

\begin{tabular}{|c|c|c|c|c|c|}
\hline Sistema & Eluente & Adsorvente & Marcadores & $h R_{f} c$ & Janela de busca \\
\hline \multirow[t]{4}{*}{ CCD 1} & \multirow[t]{4}{*}{ Clorofórmio:Acetona $(80+20)$} & \multirow[t]{4}{*}{ Sílica } & Paracetamol & 15 & \multirow[t]{4}{*}{7} \\
\hline & & & Clonazepan & 35 & \\
\hline & & & Secobarbital & 55 & \\
\hline & & & Metil-fenobarbital & 70 & \\
\hline \multirow[t]{4}{*}{$\mathrm{CCD} 2$} & \multirow[t]{4}{*}{ Acetato de Etila } & \multirow[t]{4}{*}{ Sílica } & Sulfatiazol & 20 & \multirow[t]{4}{*}{8} \\
\hline & & & Fenacetina & 38 & \\
\hline & & & Salicilamida & 55 & \\
\hline & & & Secobarbital & 68 & \\
\hline \multirow[t]{4}{*}{ CCD 3} & \multirow[t]{4}{*}{ Clorofórmio:Metanol $(90+10)$} & \multirow[t]{4}{*}{ Sílica } & Hidroclorotiazida & 11 & \multirow[t]{4}{*}{8} \\
\hline & & & Sulfafurazol & 33 & \\
\hline & & & Fenacetina & 52 & \\
\hline & & & Prazepan & 72 & \\
\hline \multirow[t]{4}{*}{$\mathrm{CCD} 4$} & Acetato de Etila:Metanol: & \multirow[t]{4}{*}{ Sílica } & Atenolol & 22 & \multirow[t]{4}{*}{8} \\
\hline & \multirow[t]{3}{*}{ Amônia $(85+10+5)$} & & Codeína & 35 & \\
\hline & & & Carbamazepina & 56 & \\
\hline & & & Diazepam & 76 & \\
\hline \multirow[t]{4}{*}{ CCD 5} & \multirow[t]{4}{*}{ Metanol } & \multirow[t]{4}{*}{ Sílica } & Codeína & 20 & \multirow[t]{4}{*}{9} \\
\hline & & & Trimipramina & 36 & \\
\hline & & & Hidroxizina & 56 & \\
\hline & & & Diazepam & 82 & \\
\hline \multirow[t]{4}{*}{ CCD 6} & Metanol:n-butanol $(60+40)$ & \multirow[t]{4}{*}{ Sílica } & Codeína & 22 & \multirow[t]{4}{*}{9} \\
\hline & \multirow[t]{3}{*}{$\mathrm{NaBr} 0,1 \mathrm{M}$} & & Difenilhidramina & 48 & \\
\hline & & & Quinina & 65 & \\
\hline & & & Diazepam & 85 & \\
\hline \multirow[t]{4}{*}{$\mathrm{CCD} 7$} & \multirow[t]{4}{*}{ Metanol:Amônia $(100$ + 1,5) } & Sílica impregnada & Atropina & 18 & 8 \\
\hline & & $\mathrm{c} / \mathrm{KOH} 0,1 \mathrm{M}$ e seca & Codeína & 33 & \\
\hline & & & Cloroprotixeno & 56 & \\
\hline & & & Diazepam & 75 & \\
\hline CCD 8 & Cicloexano:tolueno:dietilamina & Sílica impregnada & Codeína & 6 & 11 \\
\hline & $(75+15+10)$ & c/ $\mathrm{KOH} 0,1 \mathrm{M}$ e seca & Desipramina & 20 & \\
\hline & & & Prazepan & 36 & \\
\hline & & & Trimipramina & 62 & \\
\hline CCD 9 & Clorofórmio:Metanol $(90+10)$ & Sílica impregnada & Desipramina & 11 & 11 \\
\hline & & $\mathrm{c} / \mathrm{KOH} 0,1 \mathrm{M}$ e seca & Fisostigmina & 36 & \\
\hline & & & Trimipramina & 54 & \\
\hline & & & Lidocaína & 71 & \\
\hline CCD 10 & Acetona & Sílica impregnada & Amitriptilina & 15 & 9 \\
\hline & & $\mathrm{c} / \mathrm{KOH} 0,1 \mathrm{M}$ e seca & Procaína & 30 & \\
\hline & & & Papaverina & 47 & \\
\hline & & & Cinarizina & 65 & \\
\hline
\end{tabular}

Uma forma de aumentar a capacidade de identificação em CCD é a utilização de um sistema de identificação com alguma capacidade discriminante. Uma alternativa simples é a utilização de reações de cor, devidamente codificadas. Nesta abordagem, as placas cromatográficas são submetidas a reações de cor seqüenciais com registro das cores obtidas em cada estágio, as quais são comparadas com aquelas presentes na base de dados. Assim, além do valor de $\mathrm{hR}_{\mathrm{f}} \mathrm{c}$, as substâncias também podem ser classificadas com base nas cores obtidas. As cores obtidas após o processo de revelação são classificadas em 10 códigos, a saber: 0 para branca, 1 para amarela, 2 para laranja, 3 para marrom, 4 para vermelha, 5 para violeta, 6 para preta, 7 para azul, 8 para azul-esverdeada e 9 para verde. Esta metodologia permite ampliar substancialmente o poder de identificação em $\mathrm{CCD}^{30}$.

A seqüência de reações de cor propostas por Hegge e colaboradores ${ }^{30}$ é expor a vapores de formaldeído $37 \%$ e imergir ou borrifar com reagente de Mandelin (metavanadato de amônio 0,1\% em ácido sulfúrico concentrado); imergir em água; observar sob radiação UV com comprimento de onda de $366 \mathrm{~nm}$ e imergir ou borrifar com reagente de Dragendorff (subnitrato de bismuto, iodo e iodeto de potássio em meio ácido). Esta sequiência de revelação deve detectar compostos com grande diversidade funcional, como alcalóides, anfetaminas, opiáceos e outros de interesse toxicológico. Após cada estágio da sequiência de revelação, as cores são classificadas em uma escala de 0 a 9.

\section{DETECÇÃO E DIFERENCIAÇÃO POR CROMATOGRAFIA GASOSA}

A cromatografia gasosa tem sido amplamente empregada em triagem toxicológica por sua elevada capacidade de separação e reprodutibilidade. $\mathrm{O}$ parâmetro de identificação deve ser um valor relativamente independente das condições cromatográficas, exceto da natureza da fase estacionária e da própria substância. O parâmetro mais reprodutível interlaboratorialmente e, portanto, mais adequado para utilização em bases de dados é o índice de retenção linear $\left(I^{\mathrm{T}}\right)$, segundo Kovats ${ }^{1,4}$. 
No caso da análise toxicológica sistemática por CG, são obtidas curvas com valores de $I^{\mathrm{T}}$ tabelados dos fármacos utilizados como referência contra seus tempos de retenção obtidos no sistema padronizado, empregando-se temperatura programada e colunas capilares. Sob essas condições, obtém-se um gráfico quase linear, sendo o $I^{\mathrm{T}}$ de uma substância desconhecida determinado por interpolação linear entre duas substâncias de referência adjacentes ou através da Equação $4^{4,31}$.

$I^{\mathrm{T}}=\left(t_{x}-t_{0}\right) \cdot \frac{\left(I^{\mathrm{T}}{ }_{n+1}-I^{\mathrm{T}}{ }_{n}\right)}{\left(t_{n+1}-t_{n}\right)}+I^{\mathrm{T}}{ }_{n}$

onde: $\mathrm{t}_{\mathrm{x}}$ é o tempo de retenção da substância $\mathrm{X}, \mathrm{t}_{0}$ é o tempo de retenção de uma substância não retida pela coluna cromatográfica, $\mathrm{t}_{\mathrm{n}}$ e $\mathrm{t}_{\mathrm{n}+1}$ são os tempos de retenção dos marcadores que eluem antes e depois do analito, e $I^{\mathrm{T}}{ }_{\mathrm{n}}$, e $I^{\mathrm{T}}{ }_{\mathrm{n}+1}$ são os índices de retenção lineares dos marcadores que eluem antes e depois do analito.

A TIAFT também publicou uma base de dados de índices de retenção de mais de 4000 substâncias, obtidas em colunas capilares com fase estacionária de dimetilsilicone ${ }^{4}$. Para a utilização dos dados desta base é importante reproduzir fielmente a metodologia empregada, especialmente com respeito à programação de temperatura. As condições cromatográficas recomendadas utilizam coluna dimetilsilicone com $10-15$ m de comprimento com 0,32 ou 0,53 $\mathrm{mm}$ de d. i. e programação de temperatura variando entre $135 \mathrm{e}$ $312{ }^{\circ} \mathrm{C}^{4,31}$. Para o cálculo dos índices de retenção linear são utilizadas misturas contendo 10-15 fármacos, denominados marcadores de retenção, com tempos de retenção dispersos ao longo do tempo de duração da análise ${ }^{4}$.

Adicionalmente, também foi gerada uma biblioteca de índices de retenção de solventes e outras substâncias voláteis em um sistema padronizado de CG, por Machata ${ }^{32}$.

Os valores de $I^{\mathrm{T}}$ calculados para as substâncias desconhecidas empregando-se as misturas de drogas sugeridas são comparados com os valores de $I^{\mathrm{T}}$ tabelados, utilizando-se uma janela de busca de aproximadamente 20-25 unidades de $I^{\mathrm{T}}$ 2,4.

\section{DIFERENCIAÇÃO-DETECÇÃO POR CROMATOGRAFIA LÍQUIDA DE ALTA EFICIÊNCIA}

Através da CLAE é possível analisar compostos distribuídos em uma ampla faixa de polaridade, massa molecular e labilidade térmica. A compatibilidade da fase móvel com as matrizes biológicas aquosas faz a preparação da amostra mais simples, rápida e, por conseqüência, facilmente automatizável. Por outro lado, a resolução da CLAE é geralmente baixa quando comparada com a CG e por isso o poder de identificação da CLAE, utilizando somente parâmetros de retenção, é considerado levemente inferior à associação de análises, em paralelo, por CG e espectroscopia UV. Contudo, a baixa seletividade do UV pode ser utilmente explorada em ATS para a detecção de metabólitos, que freqüentemente mantêm características espectrais semelhantes aos compostos originais ${ }^{10,33,34}$. A utilização de CLAE em fase reversa e eluição por gradiente é a estratégia mais freqüentemente adotada em ATS, possibilitando a separação de um grande número de compostos com diferentes características físico-químicas, em um único desenvolvimento cromatográfico $^{34}$. Entretanto, a eluição isocrática tem suas vantagens visto que o comportamento de retenção é mais reprodutível e o "background", devido à contribuição espectral da fase móvel, é constante ao longo da corrida, otimizando o processo de quantificação. Assim, abordagens alternativas à eluição por gradiente têm sido adotadas, com a utilização de combinações de sistemas isocráticos para permitir a identificação de substâncias de características físico-químicas bastante diversas ${ }^{10,33,34}$.
Com a utilização de detectores de arranjo de diodos (DAD), a estratégia mais comum é usar os dados de retenção como um préfiltro de possíveis candidatos, submetendo os compostos que eluem em uma determinada janela de tempo a uma busca em biblioteca espectral. As rotinas de comparação espectral normalmente são precedidas da avaliação da pureza espectral dos picos ${ }^{10,33-36}$.

Uma alternativa para gerar dados comparáveis interlaboratorialmente em CLAE é a utilização de índices de retenção line$\operatorname{ar}^{11,12}$. O conceito de índices de retenção, primeiramente desenvolvido para sistemas de CG, também foi aplicado em CLAE. Diversas séries homólogas de compostos foram avaliadas como escalas de índices de retenção para CLAE-UV, especialmente alcan-2-onas, alquilarilcetonas e 1-nitroalcanos. A escala de 1-nitroalcanos demonstrou algumas vantagens. Os homólogos menores, ao contrário das alquilarilcetonas, têm fatores de retenção similares a fármacos polares, são menos reativos e possuem maior absorbância que as alcan-2-onas. A variabilidade dos $I^{\mathrm{T}}$, em condições de gradiente, é muito menor que outros parâmetros de retenção. Assim, o conceito de índices de retenção corrigidos foi introduzido e aplicado inicialmente para uma escala de alquiarilcetonas e, posteriormente, para a escala de 1-nitroalcanos ${ }^{11}$. Nesta abordagem, assume-se que as diferenças nos valores de $I^{\mathrm{T}}$ obtidos pelas mesmas substâncias em diferentes colunas podem ser normalizadas pelo escalonamento dos resultados, usando padrões de fármacos de referência com índices de retenção conhecidos. Duas escalas são utilizadas, uma para compostos ácidos e neutros e outra para compostos básicos. Assume-se que a reprodutibilidade destes $I^{\mathrm{T}}$ é constante em toda a faixa, permitindo o cálculo de índices de similaridade $2,11,12,31$. Analogamente ao procedimento utilizado em CG, são empregadas misturas contendo 6 a 8 fármacos como marcadores de retenção. A janela de busca usual é de cerca de 8 unidades de $I^{\mathrm{T}}{ }^{11,12}$.

Também foram publicados diversos trabalhos utilizando bases de dados de retenção cromatográfica em CLAE baseadas em tempo de retenção relativo $(\mathrm{r})$. O r é definido como a razão entre o tempo de retenção ajustado de um composto e o tempo de retenção ajustado de uma substância de referência, nas mesmas condições analíticas ${ }^{10}$.

Bogusz e $\mathrm{Wu}^{11}$ e Pragst e colaboradore ${ }^{10}$ publicaram abrangentes bases de dados de $I^{\mathrm{T}}$ e $\mathrm{r}$, respectivamente, para análise toxicológica por CLAE. Bogusz e $\mathrm{Wu}^{11}$ recomendaram um sistema de CLAE com eluição por gradiente utilizando diferentes proporções de acetonitrila e tampão fosfato trietilamônio $\mathrm{pH} 3,0$, em colunas de fase reversa, a partir do qual foi gerada uma coleção com cerca de 800 índices de retenção. Pragst e colaboradores ${ }^{10}$ empregaram uma fase móvel composta de mistura de acetonitrila e tampão fosfato pH 3,0 (37:63 v/v) com fase estacionária octilsílica. Desta forma, foi desenvolvida uma biblioteca de tempos de retenção relativos, bem como de espectros de ultravioleta na faixa de 195 a $380 \mathrm{~nm}$ para mais de 2600 compostos.

\section{IDENTIFICAÇÃO DOS ANALITOS}

Após a etapa de obtenção de dados analíticos, que compreende preparação da amostra, seleção dos sistemas analíticos apropriados e realização das análises, procede-se ao tratamento dos dados. Este tratamento inicia-se pelo cálculo dos parâmetros de identificação corrigidos, tais como $\mathrm{hR}_{\mathrm{f}} \mathrm{c}, I^{\mathrm{T}}$ ou $\mathrm{r}$. Considerando que estes parâmetros são comparáveis interlaboratorialmente, é possível realizar a busca nas bases de dados. Utilizando um enfoque probabilístico, todas as substâncias presentes na base de dados que se encontrem dentro da janela de busca se qualificam para ser identificadas como o desconhecido, apenas com probabilidades diferentes, seguindo uma distribuição normal. Para cada uma das substâncias inseridas na faixa da janela de busca, a probabilidade é 
calculada pela Equação 5 $5^{1,2,37}$. O valor obtido, multiplicado por 100, é denominado índice de similaridade (IS).

$P\left(x_{i j}\right)=\frac{1}{s \cdot \sqrt{2 \pi}} e^{-1 / 2}\left[\left(x_{i j}-x_{j}\right) / s\right]^{[2]}$

onde: $\mathrm{x}_{\mathrm{ij}}$ é o valor do parâmetro de identificação determinado para o composto desconhecido, $x_{j}$ é o valor da substância presente na base de dados com a qual está sendo feita a comparação e s é o desviopadrão interlaboratorial do parâmetro de identificação.

Quando apenas um sistema analítico é utilizado, um grande número de substâncias se qualificarão, não permitindo uma identificação satisfatória. Alternativamente, com a utilização de múltiplos sistemas analíticos, somente compostos presentes simultaneamente nas diversas listas de substâncias-candidato apresentarão valores de IS elevados. Este fato é demonstrado na simulação apresentada na Tabela 1.

Um caso particular ocorre na CCD associada a reações de cores padronizadas. A similaridade com relação aos códigos de cores é dada pela distância entre as cores, considerando uma distribuição "circular" das cores. Neste caso, o IS $_{\mathrm{CCD}}$ é obtido pela Equação 6, com pesos diferentes para os índices de similaridade obtidos pela comparação de $\mathrm{hR}_{\mathrm{f}} \mathrm{c}$ e códigos de cores $(\mathrm{CC})^{37}$.

$I S_{C C D}=\sqrt[3]{I S_{R F} \bullet \sqrt[2]{I S_{C C 1} \bullet I S_{C C 2} \bullet I S_{C C 3} \bullet I S_{C C 4}}}$

onde: $\mathrm{IS}_{\mathrm{RF}}$ é o índice de similaridade obtido utilizando $\mathrm{hR}_{\mathrm{f}} \mathrm{c}$ e IS $\mathrm{CC}$ são os índices de similaridade utilizando os códigos de cores na sequiência de reações de cor padronizadas.

O índice de similaridade final é obtido através da média geométrica dos índices de similaridade obtidos para cada sistema analítico. Na Equação 7 é apresentado o cálculo do índice de similaridade associando dados obtidos por CCD, CG e CLAE.

$I S=\sqrt[3]{I S_{C C D} \bullet I S_{C G} \bullet I S_{C L A E}}$

onde: $\mathrm{IS}_{\mathrm{CCD}}$ é o índice de similaridade obtido através do sistema padronizado de $\mathrm{CCD}, \mathrm{IS}_{\mathrm{CG}}$ é o índice de similaridade obtido através do sistema padronizado de CG e IS $_{\mathrm{CLAE}}$ é o índice de similaridade obtido através do sistema padronizado de CLAE.

\section{APRESENTAÇÃO DO SISTEMA INFORMATIZADO PARA ANÁLISE TOXICOLÓGICA SISTEMÁTICA}

Com o propósito de permitir a realização de procedimentos de análise toxicológica sistemática de forma eficiente e produtiva, foi desenvolvido um Sistema de Análise Toxicológica Sistemática (SATS), acessível através da internet (www.feevale.br/toxicologia/ ats), que permite o cálculo dos parâmetros de retenção cromatográfica ajustados em sistemas de CCD, CG e CLAE e a busca produtiva em bases de dados de comportamento cromatográfico. Este sistema incorpora bases de dados previamente publicadas, que incluem 10 sistemas de CCD (nomeados CCD 1 a CCD 10) ? $^{3}, 1$ sistema de reações de cor codificadas (nomeado $\mathrm{CC})^{3}, 1$ sistema de $\mathrm{CG}$ (nomeado CG 1 - IR) ${ }^{4}$ e 2 sistemas de CLAE (nomeados CLAE 1 - IR e CLAE 2 - TRR) $)^{10,11}$.

O SATS foi desenhado e construído para a plataforma windows (Windows Server, SQL Server, Internet Information Services e Active Server Pages - ASP). A construção do sistema seguiu modelos de desenvolvimento consolidados onde o banco de dados se apresenta no formato cliente/servidor (SQL Server). O processamento utiliza procedimentos armazenados no banco de dados e o ASP implementa a interface e parte do processamento.
O uso do SATS é gratuito, permitindo a geração de uma senha individual após o cadastramento do usuário na opção login/novo, na barra de opções superior. Usuários cadastrados também podem realizar buscas sobre os dados armazenados no sistema referente a uma dada substância utilizando a opção busca na barra de opções superior, bem como consultar os resultados de suas análises anteriores.

Para o processamento dos dados de uma análise, o usuário deve selecionar inicia análise na barra de opções superior. Após o cadastramento da análise, são apresentados os sistemas analíticos disponíveis para seleção (Figura 1). Selecionando a opção avançar são apresentadas as telas de entrada de dados para cada sistema analítico previamente selecionado, onde são inseridos os dados obtidos para os marcadores e para os desconhecidos. $\mathrm{O}$ usuário também pode modificar os valores das janelas de busca. A Figura 2 apresenta a tela de entrada de dados para o sistema CCD 4, e a Figura 4 apresenta a tela de entrada de dados para o sistema CLAE 2 - TRR. Após a conclusão da entrada dos dados e a seleção da opção finaliza, é apresentada uma lista de substâncias candidato com seus respectivos índices de similaridade, conforme a Figura 5. Utilizando o nome de usuário visitante@feevale.br e a senha 1234 é possível visualizar um exemplo de análise utilizando o sistema. Para tanto, é necessário selecionar a opção lista análise na barra de opções e a opção acessar ao lado da identificação das análises cadastradas.

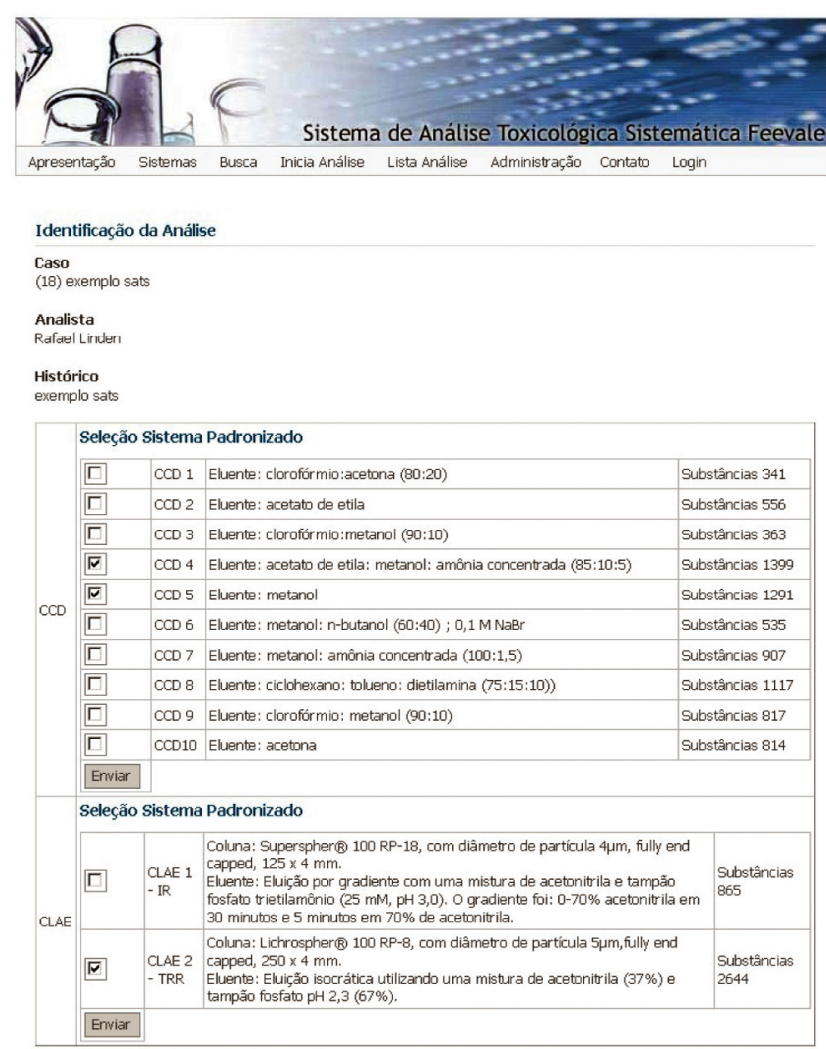

Figura 1. Tela de seleção dos sistemas analíticos disponíveis no sistema

O SATS realiza as seguintes operações com os dados fornecidos pelo usuário: a) cálculo de parâmetros de retenção corrigidos para substâncias desconhecidas para cada um dos sistemas analíticos $\left(\mathrm{hR}_{\mathrm{f}} \mathrm{c}\right.$, $I^{T}$ e r), a partir da inserção dos dados obtidos na utilização dos sistemas padronizados. O usuário deve informar os valores obtidos para os marcadores e as substâncias desconhecidas, em cada sistema empregado, conforme apresentado na Figura 2 para o sistema de CCD 4 e na Figura 4 para o sistema CLAE 2 - TRR. O usuário pode modificar os marcadores, selecionando substâncias que estejam disponíveis em seu 
laboratório, e a janela de erro, que corresponde ao desvio-padrão interlaboratorial do parâmetro de retenção multiplicado por três. Nos sistemas de CCD, o sistema também permite a inserção dos códigos de cores, selecionados em uma palheta; b) cálculo do IS para cada sistema analítico, utilizando a Equação 5; c) diferentes combinações entre os sinais analíticos encontrados. Por ex., se forem empregados três sistemas analíticos, com duas substâncias desconhecidas em cada sistema, existem 8 combinações de manchas e/ou picos que devem ser levadas em consideração; d) associações entre os IS obtidos em cada sistema analítico, calculando o IS resultante da associação dos diferentes métodos utilizando as Equações 6 e 7 ; e) apresentação da listagem de substâncias-candidato, para cada combinação possível, ordenadas por IS (Figura 5). Na tela de apresentação dos resultados também são apresentados o número de sistemas analíticos nos quais estão inseridos dados de cada substância-candidato e o número de registro da substância no "Chemical Abstracts Service"; f) definição de IS\% mínimos para apresentação dos resultados. Os valores de corte podem ser selecionados entre 5, 25, 45 e $65 \%$ e, g) definição de combinações de referência, permitindo o reprocessamento dos dados e a apresentação somente de combinações compatíveis com a referência.

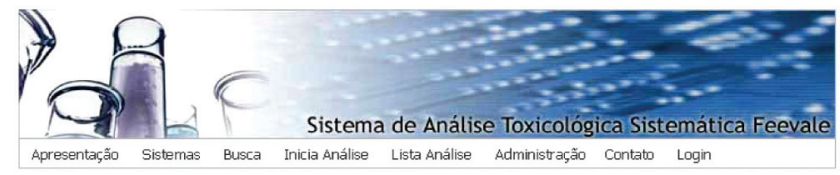

\begin{tabular}{|c|c|c|c|c|c|c|c|}
\hline $\begin{array}{l}\text { Sistema Analitico: } \\
C C D\end{array}$ & \multicolumn{4}{|c|}{$\begin{array}{l}\text { Sistema Padronizado: } \mathrm{CCD}^{4} \\
\text { Eluente: acetato de etila: metanol: amônia concentrada } \\
\text { (85:10:5) }\end{array}$} & \multicolumn{3}{|c|}{$\begin{array}{l}\text { Janela de Busca: } 10 \\
\begin{array}{|l}\text { Atualiza } \\
\end{array}\end{array}$} \\
\hline \multicolumn{8}{|l|}{ Marcadores } \\
\hline \multicolumn{4}{|l|}{ Subotância } & \multicolumn{2}{|c|}{ hRf modido } & \multicolumn{2}{|c|}{ hRfic tabolado } \\
\hline \multicolumn{2}{|l|}{$0 1 \longdiv { \text { Atenolol } ( 2 2 . 0 0 ) }$} & \multicolumn{2}{|r|}{$\nabla$} & \multicolumn{2}{|l|}{23} & \multicolumn{2}{|c|}{22} \\
\hline \multicolumn{2}{|l|}{$0 2 \longdiv { \text { Codeine } ( 3 5 . 0 0 ) }$} & \multicolumn{2}{|r|}{$\nabla$} & \multicolumn{2}{|l|}{36} & \multicolumn{2}{|l|}{35} \\
\hline \multicolumn{2}{|c|}{$0 3 \longdiv { \text { Carbamazepine( } 5 6 . 0 0 ) }$} & \multicolumn{2}{|r|}{$\nabla$} & \multicolumn{2}{|l|}{55} & \multicolumn{2}{|l|}{56} \\
\hline \multicolumn{4}{|c|}{$0 4 \longdiv { D i a z e p a m / K e t a z o l a m ~ M ~ / M e d a z e p a m ~ M ( 7 6 . 0 0 ) ~ }$} & \multicolumn{2}{|l|}{77} & \multicolumn{2}{|l|}{76} \\
\hline \multicolumn{8}{|l|}{ Atualiza } \\
\hline \multicolumn{8}{|l|}{ Desconhecidos } \\
\hline \multirow{2}{*}{\multicolumn{2}{|c|}{$\begin{array}{l}\text { Informe dados da substância } \\
\text { desconhecida }\end{array}$}} & $\begin{array}{l}\text { hRf } \\
\text { medido }\end{array}$ & Cor Medida 1 & Cor Medida 2 & Cor Medida 3 & Cor Medida 4 & $\begin{array}{l}\text { hRfi } \\
\text { calculado }\end{array}$ \\
\hline & & & In medido & ñ medido & ก medido & ก medido & Inserir \\
\hline \multicolumn{2}{|l|}{ Desconhecido \#1 } & 42 & $3 \square$ & $3 \quad \square$ & $\square$ & $\square$ & 41.63 \\
\hline \multicolumn{2}{|c|}{ Excluir Desconhecido \#2 } & 68 & 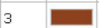 & 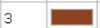 & 0 & 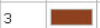 & 67.82 \\
\hline
\end{tabular}

Figura 2. Tela de entrada de dados no sistema, referente ao sistema CCD 4

\section{SIMULAÇÃO DA UTILIZAÇÃO DO SATS}

Na Tabela 2 são apresentados os dados obtidos na utilização do SATS, com dados tabelados do antidepressivo tricíclico imipramina (Figura 3), utilizando diferentes sistemas analíticos padronizados. Quando apenas o sistema CCD 4 é utilizado, mais de 50 compostos presentes na base de dados se qualificam como a substância desconhecida com similaridades superiores a $65 \%$. Ao associar-se o sistema CCD 5 aos dados previamente obtidos, apenas 6 substâncias se qualificam com IS superiores a $65 \%$. Com a adição do sistema CCD 7, apenas 2 substâncias apresentam IS superior a $65 \%$ e a adição dos códigos de cores permitiu diferenciar a imipramina de todas as outras substâncias presentes na base de dados. Também, pode-se observar que a combinação de dois métodos instrumentais, CLAE 2 - TRR e CG 1 - IR, apresenta resultados muito similares à associação de três sistemas de CCD com reações de cor seqüenciais na placa cromatográfica.

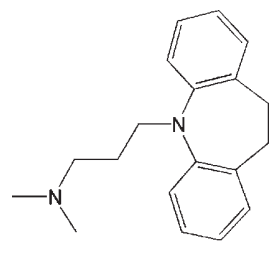

imipramina

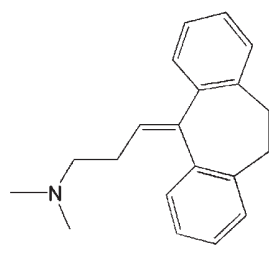

amitriptilina

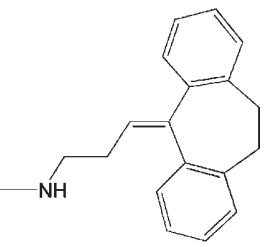

nortriptilina

Figura 3. Estruturas químicas dos antidepressivos tricíclicos imipramina, amitriptilina e nortriptilina

\section{UTILIZAÇÃO DO SATS PARA TRIAGEM TOXICOLÓGICA EM UMA AMOSTRA DE PLASMA HUMANO}

Uma amostra de plasma fortificada com os antidepressivos tricíclicos amitriptilina e nortriptilina (Figura 3), com concentrações de $5 \mu \mathrm{g} / \mathrm{mL}$, foi submetida à extração em meio alcalino $(\mathrm{pH}$ $9,0)$ com uma mistura de hexano:acetato de etila (7:3). O extrato orgânico foi levado à secura e posteriormente submetido à análise toxicológica sistemática utilizando os sistemas padronizados CCD 4 e CCD 5 (com reações de cor seqüenciais) e CLAE 2- TRR.

Após a seleção dos sistemas analíticos padronizados, o usuário deve informar os valores obtidos com os marcadores em cada sistema. Também, é possível alterar os marcadores do sistema, bem como as janelas de erro. No sistema CCD 4 foram obtidas manchas com $h R_{\mathrm{f}} \mathrm{c}$ 42 e 68, com códigos de cor 3-3-3-3 e 3-3-4-3, respectivamente. No sistema CCD5, obteve-se $\mathrm{hR}_{\mathrm{f}} \mathrm{c} 86$ e 28, com códigos de cor 3-3-3-3 e 3-3-4-3, respectivamente. No sistema CLAE 2 - TRR foram obtidos picos com tempos de retenção de 12,3 e 14,5 min. A partir da entrada

Tabela 2. Resultados obtidos no SATS, utilizando dados tabelados do antidepressivo tricíclico imipramina

\begin{tabular}{lccc}
\hline Sistemas analíticos empregados & \multicolumn{2}{c}{ Candidatos apresentados pelo SATS } \\
\hline CCD 4 & IS $>5 \%$ & $>50$ & $>50$ \\
CCD 4 + CCD 5 & $>50$ & 10 & $65 \%$ \\
CCD 4 + CCD 5 + CCD 7 & $>50$ & 5 & 2 \\
CCD 4 + CCD 5 + CCD 7 + CC & $>50$ & 1 & 1 \\
CCD 4 + CC + CG 1 -IR & 27 & 2 & 1 \\
CCD 4 + CC + CLAE 2 - TRR & 14 & 2 & 1 \\
CLAE 2 - TRR + CG 1 -IR & 27 & 1 & 1 \\
CCD 4 + CC + CLAE 2 - TRR + CG 1 -IR & 27 & 1 & 1 \\
\hline
\end{tabular}


destes dados e dos dados dos marcadores, o SATS calculou os parâmetros de retenção corrigidos $\left(\mathrm{hR}_{\mathrm{f}} \mathrm{c}\right.$ e r). Na Figura 2 é apresentada a entrada de dados para o sistema CCD 4. De forma análoga, foram lançados os dados do sistema CCD 5. A Figura 4 apresenta a interface para entrada de dados do sistema CLAE 2 - TRR.
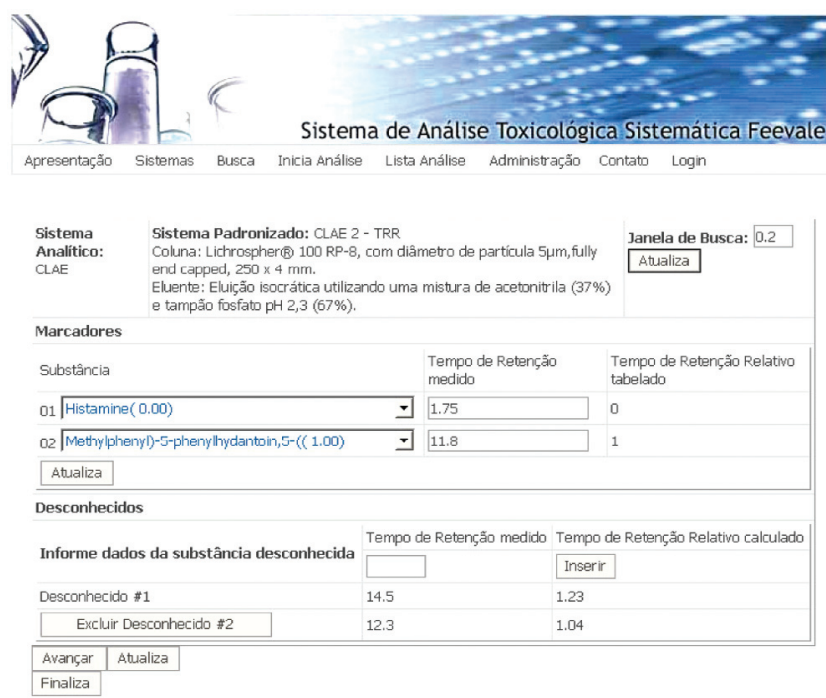

Figura 4. Tela de entrada de dados no sistema, referente ao sistema CLAE 2 - TRR

Após processamento dos dados, foi obtida uma lista de candidatos conforme a Figura 5. O SATS oferece a opção de modificar a porcentagem mínima para apresentação da relação de substânciascandidato. Neste caso, foi utilizada uma porcentagem de corte de $45 \%$. Nesta análise, considerando a existência de dois compostos, existem 8 combinações de resultados possíveis. Os compostos amitriptilina e nortriptilina apresentaram índices de similaridade
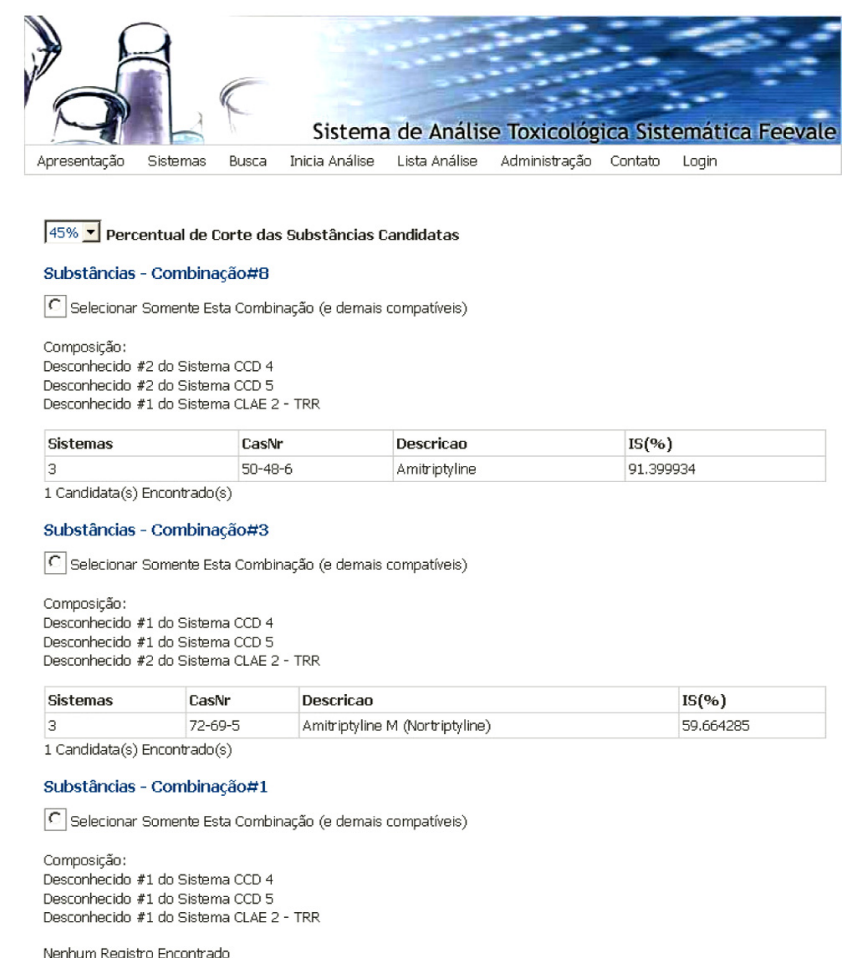

Figura 5. Tela com resultados finais da análise, apresentado as combinações possíveis e seus índices de similaridade elevados (91,4 e 59,7\%, respectivamente), com valores marcadamente superiores aos demais candidatos. Cabe salientar que as duas combinações são compatíveis.

\section{CONCLUSÕES}

A triagem toxicológica é uma das atividades fundamentais de qualquer laboratório de toxicologia clínica e forense. Uma das formas mais racionais para realização de triagens que compreendam um grande número de substâncias é a análise toxicológica sistemática. Entretanto, apesar da fundamentação científica da ATS, a sua aplicabilidade é limitada pela dificuldade associada ao tratamento dos dados obtidos e à busca simultânea em diversas bases de dados. Desta forma, a disponibilidade de um sistema informatizado para ATS representa uma alternativa valiosa para os toxicologistas analíticos. O sistema proposto, baseado em um enfoque probabilístico, permitiu identificar compostos de forma adequada, utilizando sistemas analíticos padronizados. Uma limitação natural é o universo restrito de compostos presentes na base de dados agregada ao sistema. O SATS possui propósitos educacionais, permitindo simulações variadas. Este sistema está acessível em www.feevale.br/toxicologia/ats, de forma gratuita. Uma característica vantajosa do SATS é permitir a identificação de um grande número de compostos utilizando métodos analíticos simples, mesmo com uma disponibilidade limitada de padrões analíticos. Entretanto, a efetividade deste sistema está associada à utilização de métodos estritamente padronizados, incluindo fases móveis e estacionárias e a realização de calibrações constantes.

\section{AGRADECIMENTOS}

Às valiosas sugestões do Prof. Dr. R. de Zeeuw, do University Center for Pharmacy, Groningen State University, Groningen, Holanda.

\section{REFERÊNCIAS}

1. De Zeeuw, R. A.; J. Chromatogr., B: Anal. Technol. Biomed. Life Sci. 1997, 689, 71.

2. De Zeeuw, R. A.; Hartstra, J.; Franke, J. P.; J. Chromatogr., A 1994, 674, 3.

3. De Zeeuw, R. A.; Franke, J. P.; Degel, F.; Machbert, G.; Schütz, H.; Wijsbeek, J.; Thin-Layer Chromatographic Rf Values of Toxicologically Relevant Substances on Standardized Systems; $2^{\text {nd }}$ ed., VCH Verlagsgemeinschaft: Weinheim, 1992.

4. De Zeeuw, R. A.; Franke, J. P.; Maurer, H. H.; Pfleger, K.; Gas Chromatographic Retention Índices of Toxicologically Relevant Substances on Packed or Capillary Columns with Dimethylsilicone Stationary Phases; $3^{\text {rd }}$ ed., VCH Verlagsgemeinschaft: Weinheim, 1992.

5. De Zeeuw, R. A.; J. Chromatogr., B: Anal. Technol. Biomed. Life Sci. 2004, $811,3$.

6. Hartstra, J.; Franke, J. P.; De Zeeuw, R. A.; J. Chromatogr., B: Anal. Technol. Biomed. Life Sci. 2000, 739, 125.

7. Herrin, G.; McCurdy, H. H.; Wall, W. H.; J. Anal Toxicol. 2005, 29, 599.

8. Mueller, C. A.; Weinmann, W.; Dresen, S.; Schreiber, A.; Gergov, M.; Rapid Commun. Mass Spectrom. 2005, 19, 1332.

9. Ojanpera, I.; Pelander, A.; Laks, S.; Gergov, M.; Vuori, E.; Witt, M.; J. Anal. Toxicol. 2005, 29, 34.

10. Pragst, F.; Herzler, M.; Herre, S.; Erxleben, B-T; Rothe, M.; UV Spectra of Toxic Compounds. Database of Photodiode Array UV Spectra of Illegal and Therapeutic Drugs, Pesticides, Ecotoxic Substances and Other Poisons, Dr. Dieter Helm: Heppenheim, 2001.

11. Bogusz, M.; Wu, M.; J. Anal. Toxicol. 1991, 15, 188.

12. Bogusz, M.; Erkens, M.; J. Chromatogr., A 1994, 674, 97.

13. Boukhabza, A.; Lugnier, A. A. J.; Kintz, P.; Mangin, P.; J. Anal. Toxicol. 1991, 15, 319

14. Drummer, O. H.; Kotsos, A.; McIntyre, I. M.; J. Anal. Toxicol. 1993, 17, 225

15. Elliot, S. P.; Hale, K. A.; J. Chromatogr., B: Anal. Technol. Biomed. Life Sci. 1994, 694, 99.

16. Elliot, S. P.; Hale, K. A.; J. Anal. Toxicol. 1998, 22, 279. 
17. Gaillard, Y.; Pépin, G.; J. Chromatogr., B: Anal. Technol. Biomed. Life Sci 1997, 763, 149.

18. Hill, D. W.; Kind, A. J.; J. Anal. Toxicol. 1994, 18, 233.

19. Lambert, W. E.; Meyer, E.; De Leenheer, A. P.; J. Anal. Toxicol. 1995, 19, 73.

20. Lambert, W. E.; Meyer, E.; Xue-Ping, Y.; De Leenheer, A. P.; J. Anal. Toxicol. 1995, 19, 35

21. Lambert, W. E.; van Bocxlaer, J. F.; De Leenheer, A. P.; J. Chromatogr. B: Anal. Technol. Biomed. Life Sci. 1997, 689, 45.

22. Logan, B. K.; Stafford, D. T.; Tebbet, I. R.; Moore, C. M.; J. Anal. Toxicol. 1990, 14, 144.

23. Schepers, P. G. A. M.; Franke, J. P.; de Zeeuw, R. A. J. Anal. Toxicol. 1983, 7, 272.

24. Maier, R. D.; Bogusz, M.; J. Anal. Toxicol. 1995, 19, 79

25. Galanos, D. S.; Kapoulas, V. M.; J. Chromatogr. 1964, 13, 128.

26. Bogusz, M.; Klys, M.; Wijsbeek, J.; Franke, J. P.; De Zeeuw, R. A.; J. Anal. Toxicol. 1984, 8, 149 .

27. Bogusz, M.; Franke, J. P.; Wijsbeek, J.; De Zeeuw, R. A.; J. Anal. Toxicol. 1986, 10,245 .
28. De Zeeuw, R. A.; Franke, J. P.; van Halem, M.; Schaapman, S.; Logawa, E.; Siregar, C. J. P.; J. Chromatogr., A 1994, 664, 263.

29. De Zeeuw, R. A.; Franke, J. P.; Dik, E.; Dollen, W. T.; Kam. B. L. J.; Forensic Sci. 1992, 37, 984.

30. Hegge, H. F. S.; Franke, J. P.; De Zeeuw, R. A.; J. Forensic Sci. 1991, 36, 1094.

31. Cantos, M.; Hikichi, N.; Rev. Bras. Toxicol. 1999, 12, 79.

32. Machata, G.; Gas Chromatographic Retention Indices of Solvents and other Volatile Substances for use in Toxicological Analysis, $\mathrm{VCH}$ Verlagsgemeinschaft: Weinheim, 1992.

33. Drummer, O. H.; J. Chromatogr., B: Anal. Technol. Biomed. Life Sci. 1999, 733, 27.

34. Polettini, A.; J. Chromatogr., B: Anal. Technol. Biomed. Life Sci. 1999, 733, 47.

35. Tracqui, A.; Kintz, P.; Mangin, P.; J. Forensic Sci. 1995, 40, 254.

36. Külpmann, W. R., ed.; Klinisch-toxicologische Analytik, Wiley-VCH Verlag: Weinheim, 2002

37. Hartstra, J.; Tese de Doutorado, Groningen State University, Holanda, 1997. 